\title{
Grid Supporting VSCs in Power Systems with Varying Inertia and Short-Circuit Capacity
}

Misyris, Georgios; Mermet Guyennet, Jeanne Anais; Chatzivasileiadis, Spyros; Weckesser, Johannes Tilman Gabriel

\section{Published in:}

Proceedings of IEEE PES PowerTech 2019

Link to article, DOI:

10.1109/PTC.2019.8810979

Publication date:

2020

Document Version

Peer reviewed version

Link back to DTU Orbit

Citation (APA):

Misyris, G., Mermet Guyennet, J. A., Chatzivasileiadis, S., \& Weckesser, J. T. G. (2020). Grid Supporting VSCs in Power Systems with Varying Inertia and Short-Circuit Capacity. In Proceedings of IEEE PES PowerTech 2019 IEEE. https://doi.org/10.1109/PTC.2019.8810979

\section{General rights}

Copyright and moral rights for the publications made accessible in the public portal are retained by the authors and/or other copyright owners and it is a condition of accessing publications that users recognise and abide by the legal requirements associated with these rights.

- Users may download and print one copy of any publication from the public portal for the purpose of private study or research.

- You may not further distribute the material or use it for any profit-making activity or commercial gain

- You may freely distribute the URL identifying the publication in the public portal 


\title{
Grid Supporting VSCs in Power Systems with Varying Inertia and Short-Circuit Capacity
}

\author{
George S. Misyris, Student Member, IEEE, Jeanne A. Mermet-Guyennet, Student Member, IEEE, \\ Spyros Chatzivasileiadis, Senior Member, IEEE, and Tilman Weckesser, Member, IEEE
}

\begin{abstract}
Voltage Source Converters (VSCs) can offer various control strategies to enable realization of the vision of a "Global Grid". With the increasing penetration of renewable energy sources, it is becoming more frequent for VSCs to be required to provide active and reactive power regulation. In this paper, a model of a grid-supporting VSC connected to a weak AC grid with low Short Circuit Capacity (SCC) and low inertia is presented. The impact of SCC and inertia is then illustrated by means of small-signal stability, $H_{2}$ norm and large-disturbance analysis.
\end{abstract}

Index Terms-High Voltage Direct Current (HVDC), stability analysis, Grid-Supporting Voltage Source Converter, weak AC grid, varying inertia.

\section{INTRODUCTION}

Due to national and global climate targets aiming at reducing the emission of greenhouse gases [1], Renewable Energy Sources (RES) progressively replace conventional generation based on fossil fuels. Increased penetration of RES adds significant uncertainty to the dynamic behaviour of power systems. This originates from the fluctuating nature of resources such as wind and solar as well as from the fact that RES are often connected through power electronics to the grid. As a result, system parameters such as inertia and Short-Circuit Capacity (SCC) will vary greatly over time, which will affect the strength and stability of (Alternating Current) AC [2].

Additionally, High Voltage Direct Current (HVDC) connections make their way against installation of new AC lines [3]. These HVDC connections are based either on Line Commutated Converters (LCCs) or Voltage Source Converters (VSCs) [4]. However, VSCs have been gaining momentum, because of their flexibility and control capabilities [5]. Consequently, they are ideally suited to integrate the growing capacity of RES being installed.

To ensure stable grid operation in the absence of conventional generators, grid-connected VSCs should participate in the regulation of the AC grid voltage and frequency, by controlling the active and reactive power delivered to the grid. These VSCs are classified as grid-supporting converters and their outer controllers are commonly equipped with droop control algorithms $(P / f$ and $Q / V$ droop characteristic) to avoid communication-based solutions [6].

By taking advantage of the ability of power electronics to respond fast using a P-f droop characteristic for HVDCVSC interconnections becomes a feasible solution to provide

G. S. Misyris, J. A. Mermet-Guyennet, S. Chatzivasileiadis and T. Weckesser are with the Department of Electrical Engineering, Technical University of Denmark, 2800 Kgs. Lyngby, Denmark (e-mail: \{gmisy, jamg, spchatz,jtgw\}@elektro.dtu.dk). frequency support in AC systems with low inertia. It has been demonstrated that this is an efficient approach for reducing the maximum frequency deviation in such systems [7], [8]. In [7], a review of supplementary local control strategies for VSCHVDC for low-inertia and islanded systems is presented. In [9], In [8], a frequency control scheme for VSCs, which are part of an HVDC grid connecting asynchronous AC systems, is proposed. In these studies, time domain simulations were used to verify the impact of the $P-f$ droop characteristic of the VSC on frequency stability. However, by time domain simulation analysis, it does not become analytically evident, how the VSC control gains contribute to AC frequency regulation. As indicated in [10], $\mathrm{H}_{2}$ system norm can be incorporated to provide a measure of the magnitude of the system output in response to a disturbance. Therefore, in this work the $\mathrm{H}_{2}$ norm is considered as a performance metric to validate the impact of the VSC control parameters on inertial and primary frequency response.

Apart from low inertia, the system can be subject to low SCC, which is dependent on the system strength. In [11], the small-signal stability of a VSC connected to weak AC system is studied, where the results show that the maximum power transfer capability of the VSC-HVDC converter is affected by the PLL gains. In [12], the authors propose a grid synchronization mechanism by introducing an impedanceconditioning term in the Phase Locked Loop (PLL), to increase the range of stable power transfer of VSCs, which operate in weak grids. Furthermore, in [5], an investigation of the stability of VSC connected subject to low SCC is performed, where the impact of the various control parameters on system stability and maximum stable power transfer of VSC are studied. In the aforementioned work, VSCs operate in a gridfeeding mode, where the active and reactive power injected to the grid are kept constant.

However as mentioned above, it is envisioned that in a future grid with high penetration from inverter-based generation and transmission, it will be necessary that VSCs actively support the grid and, hence, are operated in a grid-supporting mode. This could be realized by adding $P-f$ and $Q-V$ droop characteristics, which can affect the stability of the system and the dynamic limit of the maximum power transfer capability. An investigation of this is carried out in this work.

The contribution of this paper is to provide a groundwork for understanding the nonlinear behavior of grid-supporting VSCs, connected to weak AC grids. The system model, used in this paper, is an extension of the one presented in [5]. Moreover, constraints on the magnitudes of VSC control gains 


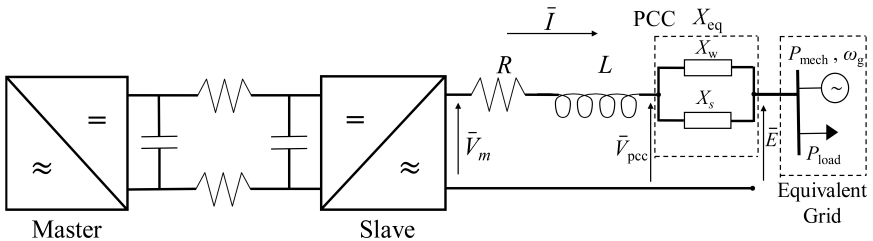

Fig. 1. Test system: VSC and equivalent grid

are defined based on eigenvalue and $H_{2}$ norm analysis. An investigation on the dynamic limit for a sudden drop of the SCC is carried out. At last, the influence of the VSC on the inertial and primary frequency response is analyzed using $\mathrm{H}_{2}$ norm and time-domain simulations.

This paper is organized as follows: Section II describes the system state space model of the system. In section III, a stability analysis of a VSC connected to weak AC system is performed. Section IV demonstrates the system response to large disturbances. Conclusions are drawn in Section VI.

\section{SySTEM Modeling}

In this section, the VSC control structure is presented, as well as the equations forming the state space model of the overall system. The state space representation relates the state variables, $x$, and the inputs of the system, $u$ to their derivatives, $\dot{x}$ and outputs of the system, $y$ in the form:

$$
\begin{aligned}
\dot{x} & =f(x, u) \\
y & =g(x, u)
\end{aligned}
$$

where $f(x, u)$ corresponds to the first order non-linear differential equations of the system, and $g(x, u)$ to the algebraic equations relating the outputs of the system to its state variables and inputs.

Fig. 1 shows the study system, i.e. two VSCs in a masterslave configuration. With this configuration, the DC link voltage $V_{\mathrm{dc}}$ is assumed to remain constant as it is controlled by the master VSC. The slave VSC controls the current flowing through the phase reactor by adjusting the voltage $V_{\mathrm{m}}$. Fig. 2 demonstrates the inner and outer control loops of the VSC.

The VSC and its components are represented in the $d q$ frame, whose $d$-axis is shifted of an angle $\theta_{\text {pll }}$ with the $x$ axis of the $x y$ frame, rotating at the speed of the Center Of Inertia (COI) $\omega_{\text {coi }}$ obtained from angular speed of the generator equivalent. $X_{\text {eq }}$ represents the Thevenin equivalent reactance of the AC grid. The equivalent reactance $X_{\text {eq }}$ can be defined as a function of SCC [11]:

$$
X_{\text {eq }}=\frac{V_{\text {rated }}^{2}}{S C C \cdot P_{\text {rated }}}
$$

where $V_{\text {rated }}$ and $P_{\text {rated }}$ are the rated AC voltage and power of the VSCs, respectively.

The AC grid is represented by a generator and a load. The bus corresponding to equivalent grid is considered as an angle reference. The corresponding equations are summarized below, all quantities being expressed in per unit, except for the angular frequencies, in rad/s. It is assumed that the magnitude of $E$ is equal to 1 p.u. and it is kept constant during the disturbances.

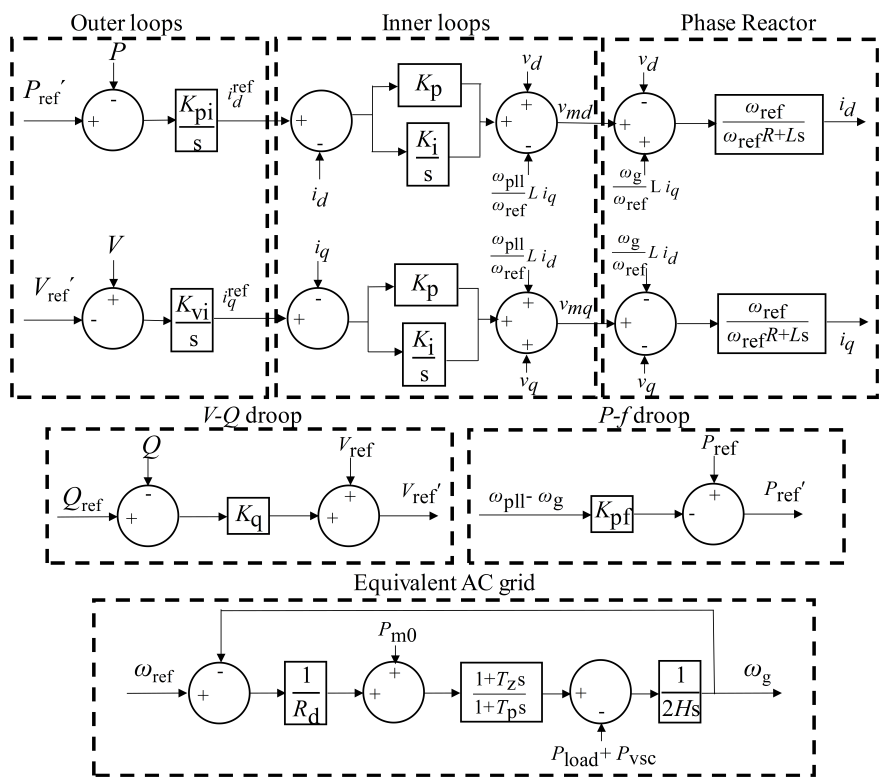

Fig. 2. Test system: VSC control

\section{A. Electromechanical dynamics of AC system}

In this section, an equivalent to represent the inertial and primary frequency response of AC system is implemented according to [13]. The purpose of the equivalent is to capture the COI frequency deviation after a power disturbance, with which the PLL is aligned. Since, a single machine equivalent is considered, $\omega_{\text {coi }}=\omega_{\mathrm{g}}$, where $\omega_{\mathrm{g}}$ corresponds to the frequency of the equivalent grid. As mentioned in [13], the current model represents primary frequency control, where power is dominantly provided by steam turbines. The nominal rotor speed is $\omega_{\mathrm{ref}}=2 \pi \cdot 50 \mathrm{rad} / \mathrm{s}$. The phase angle $\theta_{\mathrm{g}}$ of the voltage of the equivalent grid's generator is such as:

$$
\frac{d \theta_{\mathrm{g}}}{d t}=\omega_{\mathrm{ref}} \Delta \omega_{\mathrm{g}}
$$

where $\Delta \omega_{\mathrm{g}}=\omega_{\mathrm{g}}-\omega_{\text {ref. }}$. The dynamics of the frequency deviation in response of a change in electric active power seen from the equivalent grid are derived from the swing equation:

$$
\frac{d \Delta \omega_{\mathrm{g}}}{d t}=\frac{1}{M}\left(P_{\text {mech }}+P_{\mathrm{vsc}}-P_{\text {load }}\right)
$$

where $P_{\text {mech }}$ corresponds to the mechanical power input of the generator and $P_{\mathrm{vsc}}$ is the electrical power of the VSC received by the generator. The real part of the equivalent grid impedance is neglected, thus $P_{\mathrm{vsc}}=v_{d} i_{d}+v_{q} i_{q}=E_{d} i_{d}+$ $E_{q} i_{q}$, where $v_{d}, E_{d}$ and $v_{q}, E_{q}$ are the $d$ and $q$ components of $\bar{V}_{\text {pcc }}$ and $\bar{E}$, respectively. The $i_{d}$ and $i_{q}$ variables correspond to $d$ and $q$ components of the current flowing through the phase reactor and the equivalent grid impedance. The parameter $H$ is the system inertia constant given in seconds.

With a speed droop $R_{\mathrm{d}}$, and a primary frequency control similar to the one provided by steam turbines characterized by time constants $T_{\mathrm{z}}$ and $T_{\mathrm{p}}$ as described in [13], the equivalent grid mechanical power dynamics can be written as:

$$
\begin{aligned}
\frac{d P_{\text {mech }}}{d t} & =-\frac{1}{T_{\mathrm{p}}}\left(P_{\text {mech }}-P_{m 0}\right)-\frac{1}{R_{\mathrm{d}} T_{\mathrm{p}}} \Delta \omega_{\mathrm{g}} \\
& -\frac{T_{\mathrm{z}}}{2 H R_{\mathrm{d}} T_{\mathrm{p}}}\left(P_{\text {mech }}-P_{\text {load }}-P_{\mathrm{vsc}}\right)
\end{aligned}
$$




\section{B. Phase-Locked Loop (PLL)}

The VSC is synchronized with the corresponding AC grid through a PLL and aligned with the COI frequency. The PLL provides an estimation of the phase angle $\theta_{\mathrm{pll}}$ of the voltage $V_{\mathrm{pcc}}$ at the Point of Common Coupling (PCC). By adjusting $v_{q}$ with a PI controller, it aims at aligning the voltage phasor $\bar{V}_{\text {pcc }}$ with the $d$-axis of the dq rotating frame, so that in the steady-state $\omega_{\mathrm{pll}}=\omega_{\mathrm{g}}, v_{d}=V_{\text {ref }}$ and $v_{q}=0$. It can hence be represented by the following equations:

$$
\begin{aligned}
\frac{d \theta_{\mathrm{pll}}}{d t} & =\omega_{\mathrm{pll}}-\omega_{\mathrm{g}} \\
\frac{d M_{\omega}}{d t} & =K_{\mathrm{i} \omega} v_{q} \\
\omega_{\mathrm{pll}} & =K_{p \omega} v_{q}+M_{\omega}
\end{aligned}
$$

with $K_{\mathrm{i} \omega}$ and $K_{p \omega}$ the integral and proportional gains of the PI controller. $M_{\omega}$ is the integrator internal state of the PLL control system.

\section{Phase reactor}

The relations between the current $I$, the voltage output of the VSC $V_{m}$ and the voltage at the PCC $V_{\mathrm{pcc}}$ in the dq frame are as follows:

$$
\begin{aligned}
& L \frac{d i_{d}}{d t}=\omega_{\text {ref }}\left(\frac{\omega_{\mathrm{g}}}{\omega_{\text {ref }}} L i_{q}-R i_{d}+v_{m d}-v_{d}\right) \\
& L \frac{d i_{q}}{d t}=\omega_{\text {ref }}\left(-\frac{\omega_{\mathrm{g}}}{\omega_{\text {ref }}} L i_{d}-R i_{q}+v_{m q}-v_{q}\right)
\end{aligned}
$$

where $L$ is the inductance and $R$ the resistance of the phase reactor. The variables $v_{m d}$ and $v_{m q}$ correspond to the $d$ and $q$ components of voltage $\bar{V}_{m}$.

\section{Inner current control loops}

The current controller keeps the currents $i_{d}$ and $i_{q}$ to their references values, $i_{d}^{\text {ref }}$ and $i_{q}^{\text {ref }}$ by adjusting the components of the voltage of the VSC, $v_{m d}$ and $v_{m q}$ (see Fig. 2):

$$
\begin{array}{r}
v_{m d}=v_{d}-\frac{\omega_{\mathrm{pll}}}{\omega_{\mathrm{ref}}} L i_{q}+K_{\mathrm{p}}\left(i_{d}^{\mathrm{ref}}-i_{d}\right)+M_{d} \\
v_{m q}=v_{q}+\frac{\omega_{\mathrm{pll}}}{\omega_{\mathrm{ref}}} L i_{d}+K_{\mathrm{p}}\left(i_{q}^{\mathrm{ref}}-i_{q}\right)+M_{q} \\
\frac{d M_{d}}{d t}=K_{\mathrm{i}}\left(i_{d}^{\mathrm{ref}}-i_{d}\right), \quad \frac{d M_{q}}{d t}=K_{\mathrm{i}}\left(i_{q}^{\mathrm{ref}}-i_{q}\right)
\end{array}
$$

where $K_{\mathrm{p}}$ and $K_{\mathrm{i}}$ are the proportional and integral gains of the PI controllers, and $M_{d}$ and $M_{q}$ are the internal states of the integrators.

\section{E. Outer control loops}

The outer control is used to calculate the d- and q-axis current references, $i_{d}^{\text {ref }}$ and $i_{q}^{\text {ref }}$. In this current control configuration of the VSC, the active current reference $i_{d}^{\text {ref }}$ is adjusted to control the active power $P$ injected by the VSC to its reference $P_{\text {ref }}$. Due to the $P-f$ droop characteristic, the active power reference is adjusted in response to a deviation of the PLL frequency $\omega_{\text {pll }}$ with respect to the grid frequency, $\omega_{\mathrm{g}}=\omega_{\mathrm{ref}}+\Delta \omega_{\mathrm{g}}$, with $K_{\mathrm{pf}}$ the corresponding frequency droop gain. It should be noted that a deadband is considered in the range of $[49.9,50.1] \mathrm{Hz}$, to avoid continuous changes in the $\mathrm{AC}$ frequencies of the interconnected areas.

The reactive current reference $i_{q}^{\text {ref }}$ aims at keeping the voltage $V_{\text {pcc }}$ at the PCC to its reference $V_{\text {ref }}$, which is also adjusted by a $V-Q$ droop control characteristic of gain $K_{\mathrm{q}}$. The two control loops are implemented with integral controllers of gains $K_{i d}$ and $K_{i q}$ (both positive).

$$
\begin{aligned}
& \frac{d i_{d}^{\mathrm{ref}}}{d t}=K_{i d}\left(P_{\mathrm{ref}}-K_{\mathrm{pf}}\left(\omega_{\mathrm{pll}}-\omega_{\mathrm{g}}\right)-P_{\mathrm{vsc}}\right) \\
& \frac{d i_{q}^{\mathrm{ref}}}{d t}=K_{i q}\left(V_{\mathrm{pcc}}-V_{\mathrm{ref}}+K_{\mathrm{q}}\left(Q_{\mathrm{vsc}}-Q_{\mathrm{ref}}\right)\right)
\end{aligned}
$$

where $Q_{\mathrm{vsc}}=v_{q} i_{d}-v_{d} i_{q}$ and $V_{\mathrm{pcc}}=\sqrt{v_{d}^{2}+v_{q}^{2}}$

\section{F. Final state-space model}

Based on the grid equations, the $\mathrm{d}$ and $\mathrm{q}$ component of the voltage at the PCC can be written as:

$$
\begin{aligned}
& v_{d}=-X_{\mathrm{eq}} i_{q}+E \cos \left(\theta_{\mathrm{g}}-\theta_{\mathrm{pll}}\right) \\
& v_{q}=X_{\mathrm{eq}} i_{d}+E \sin \left(\theta_{\mathrm{g}}-\theta_{\mathrm{pll}}\right)
\end{aligned}
$$

where $X_{\mathrm{eq}}=X_{\mathrm{w}} \| X_{\mathrm{s}}$. After elimination of the algebraic equations describing $\omega_{\text {pll }}, v_{m d}, v_{m q}, v_{d}$ and $v_{q}$ in the above equations, we obtain a set of 11 differential equations corresponding to the vector of 11 state variables (19).

$$
x=\left[\theta_{\mathrm{pll}}, M_{\omega}, i_{d}, i_{q}, M_{d}, M_{q}, i_{d}^{\mathrm{ref}}, i_{q}^{\mathrm{ref}}, \Delta \omega_{\mathrm{g}}, \theta_{\mathrm{g}}, P_{\mathrm{mech}}\right]
$$

These differential equations form the state-space representation and can be expressed in the following way:

$$
\begin{aligned}
\frac{d x}{d t} & =A x+B_{u} u+B_{w} w \\
z & =C_{z} x
\end{aligned}
$$

where $x$ is the state vector, $w$ the disturbance vector, $u$ the input vector and $z$ the output vector. The matrices $A, B_{u}, B_{w}$ are defined based on the differential and algebraic equations (7)-(18).

Moreover, the input vector $u$ contains the variables that can be used to control the system. The disturbance vector $w$ consists of the variables that can not be used for controlling the system. At last, the vector $z$ includes the variables that can be observed and be used by the controller. The vectors $u, w$ and $z$ are given by:

$$
u=\left[P_{\text {ref }}, V_{\text {ref }}, P_{\mathrm{m} 0}, Q_{\text {ref }}\right], w=\left[P_{\text {load }}\right], z=\left[\Delta \omega_{\mathrm{g}}\right]
$$

\section{SMALL-Signal STABiLITY ANALYSIS OF VSC CONNECTED TO WEAK AC SYSTEM}

The system shown in Fig. 1 was used for the stability analysis and validation. A linearization of the system of differentialalgebraic equations is performed. The control parameters used for model validation are tuned according to [5] and shown in Table I. For the stability analysis the equivalent grid impedance is equal to $X_{\mathrm{eq}}=X_{\mathrm{w}}=2$ p.u.. 
TABLE I

CONTROL AND SYSTEM PARAMETERS

\begin{tabular}{|c|c|c|c|c|c|}
\hline Parameter & Value & Parameter & Value & Parameter & Value \\
\hline$R$ & 0.01 & $L$ & 0.2 & $M$ & 8 \\
\hline$K_{\mathrm{p}}$ & 0.2574 & $K_{\mathrm{i}}$ & 57.3 & $R_{\mathrm{d}}$ & 0.08 \\
\hline$K_{\mathrm{pi}}$ & 20 & $K_{\mathrm{vi}}$ & 15 & $T_{\mathrm{z}}$ & 1.2 \\
\hline$K_{p \omega}$ & 200 & $K_{\mathrm{i} \omega}$ & 10000 & $T_{\mathrm{p}}$ & 6.8 \\
\hline$K_{\mathrm{pf}}$ & 10 & $K_{\mathrm{q}}$ & 0.2 & $P_{\text {load }}$ & 20 \\
\hline$P_{\mathrm{m} 0}$ & 23.51 & $P_{\text {ref }}$ & 0.49 & $Q_{\text {ref }}$ & 0.4005 \\
\hline$V_{\text {ref }}$ & 1 & $X_{\mathrm{w}}$ & 2 & $X_{\mathrm{s}}$ & 0.222 \\
\hline
\end{tabular}
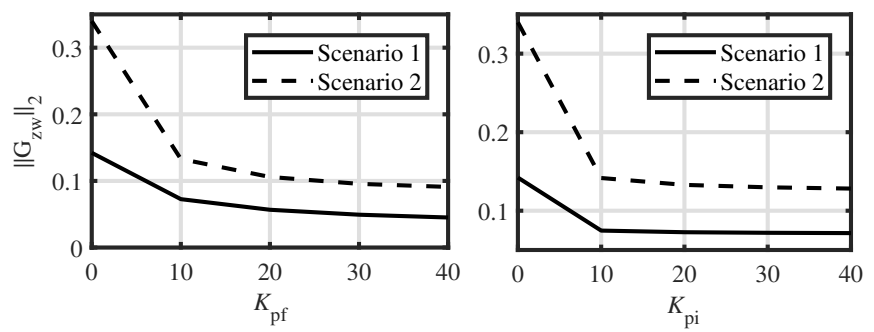

Fig. 3. Impact of gains on the $H_{2}$ norm of $G_{z w}$ (s)

\section{A. Impact of VSC control gains on inertial and primary frequency response}

To investigate the impact of VSC control gains on inertial and primary frequency response, the transfer function $G_{z w}(\mathrm{~s})$ is considered, which is relating a mismatch in active power to speed deviation $\Delta_{\omega_{\mathrm{g}}}$.

$$
G_{z w}(\mathrm{~s})=\frac{\Delta \omega_{\mathrm{g}}}{\Delta P_{\text {load }}}=C_{z}(s I-A)^{-1} B_{w}
$$

where $C_{z}$ is a matrix mapping the speed deviation of the generator equivalent. The matrices $A$ and $B_{w}$ (see (20)) are extracted by linearizing the system around an equilibrium. As defined in [10], the $H_{2}$ system norm is used as a performance metric, to measure the impact of the control gains on the AC frequency deviation. $H_{2}$ system norm takes the form of an input-output gain, which gives a measure of the total energy of the output after an instantaneous change of the input. Considering $G_{z w}(\mathrm{~s})$, the $H_{2}$ norm shows how much the rotor speed deviates from its reference value after a load disturbance $\Delta P_{\text {load }}$. Among the control gains, the frequency droop gain $K_{\mathrm{pf}}$ and the control gain of active power loop $K_{\mathrm{pi}}$ are the ones that determine the speed response of the VSC to a power imbalance [8]. To evaluate the impact of the gains, two scenarios are considered:

- Scenario $1, H=4 \mathrm{~s}, R_{\mathrm{d}}=0.08$

- Scenario $2, H=1.5 \mathrm{~s}, R_{\mathrm{d}}=0.16$

As shown in Fig. 3, both the active power loop and frequency droop gain $\left(K_{\mathrm{pi}}\right.$ and $\left.K_{\mathrm{pf}}\right)$ affect the energy of the frequency signal to a load disturbance. Increase of the frequency droop gain, decreases the $\mathrm{H}_{2}$ norm significantly. As a result, for larger values of the frequency droop, the maximum frequency deviation will be smaller after a change of the load. However, large values of $K_{\mathrm{pf}}$ can cause frequency instability for different primary frequency control schemes (hydro) [14], create large frequency dip in the other AC-system and lead to undesired dip of the DC voltage. Thus, large values for $K_{\mathrm{pf}}$ gain should be avoided.

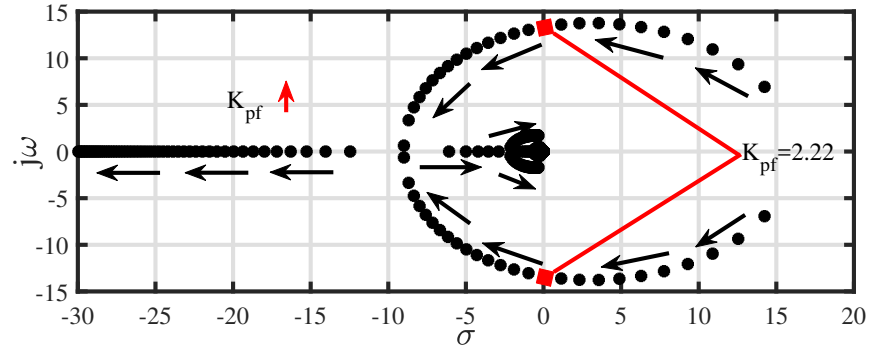

Fig. 4. Effect on system eigenvalues when varying $K_{\mathrm{pf}}$

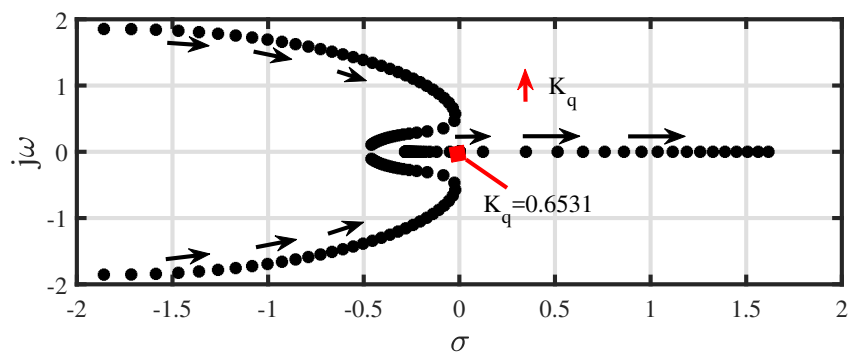

Fig. 5. Effect on dominant system eigenvalues when varying $K_{\mathrm{q}}$

Regarding the active power loop gain $K_{\mathrm{pi}}$, it is shown in Fig. 3, that for $K_{\mathrm{pi}}>20$, its impact on the frequency stability is only slightly improved. Considering the results derived in [5], accelerating the active power control deteriorates the small-signal stability of the system. Therefore, an intermediate value could be considered for the active power loop gain $K_{\mathrm{pi}}$, given that the impact on $\mathrm{H}_{2}$ norm is insignificant for higher values of it. Based on the analysis, a $K_{\mathrm{pi}}=20$ is used in the rest of the paper.

\section{B. Effect of frequency droop}

The trajectory of the system eigenvalues when varying the frequency droop gain is shown in Fig. 4. For this case, the power operating point is equal to $P_{\text {ref }}=0.49$ p.u. The value of $K_{\mathrm{pf}}$ varies in the range of [0,20]. Increasing the droop gain improves the small signal stability by moving the system eigenvalues further to the left half plane. This can be explained as follows. Following a small disturbance, a change in PCC angle occurs. The angle starts oscillating, until it reaches a new equilibrium. By adding frequency droop, the active current $i_{d}$ is quickly adjusted, the oscillations are damped out within a short time (see also Fig. 6) and the VSC reaches its new equilibrium. Based on these findings, a $K_{\mathrm{pf}}=10$ is used in the rest of the paper.

\section{Effect of $Q-V$ droop characteristic}

The root locus of the system eigenvalues, when the voltage droop gain increases, is depicted in Fig. 5. The value of $K_{\mathrm{q}}$ varies in the range of $[0,1]$. Unlike the effect of frequency droop on small-signal stability, when the voltage droop gain increases, the small-signal stability deteriorates. For $K_{\mathrm{q}}>0.6531$, the system eigenvalues become positive, thus the system becomes unstable. A large value of $K_{\mathrm{q}}$ results in prioritization of Q-control after a contingency. The higher the value of $K_{\mathrm{q}}$, the larger the voltage depression at post-fault state becomes. Thus, the maximum power transfer capability decreases significantly (24). Based on the analysis, an adequate value for the $Q-V$ droop is $K_{\mathrm{q}}=0.2$. 


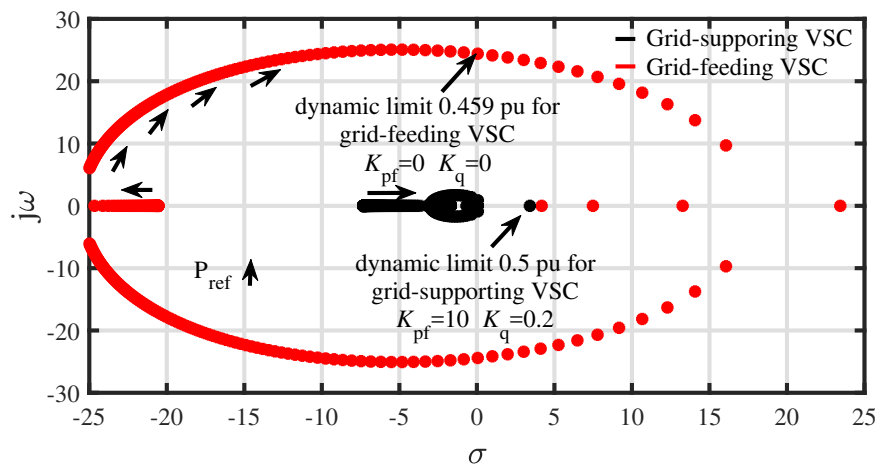

Fig. 6. Effect on dominant system eigenvalues when $P_{\text {ref }}$ increases

\section{Effect of operating point}

For low SCC, the operating point of the VSC is bounded by the static stability limit defined by:

$$
P_{\max }^{\text {static }}=\frac{V_{\mathrm{pcc}} E}{X_{\mathrm{eq}}}
$$

where $V_{\mathrm{pcc}}$ is the voltage at the PCC, $E$ is the voltage magnitude of the AC system. The impact of increasing the $P_{\text {ref }}$ of the VSC on the system eigenvalues is depicted in Fig. 6. It can be seen, that as $P_{\text {ref }}$ of the VSC increases the system eigenvalues move towards the right half plane. The real part of the eigenvalues is negative, when $P_{\text {ref }}$ is smaller than $P_{\max }^{\text {static }}$ and become positive for $P_{\text {ref }} \geq 0.5$ p.u., which results in the VSC becoming unstable. As shown in Fig. 6, the dynamic limit of grid-supporting VSC is higher than the one of gridfeeding. It will be seen later in the paper, that this happens, because of the additional frequency droop gain.

\section{LARGE Disturbance: CASE STUdies}

\section{A. Case 1 - Tripping of short reactance without fault}

The considered disturbance is a line opening without a fault and occuring at $t=0.2 \mathrm{~s}$. This increases the grid impedance from $X_{\mathrm{eq}}=X_{\mathrm{w}} \| X_{\mathrm{s}}=0.2$ p.u. to $X_{\mathrm{eq}}=X_{\mathrm{w}}=2$ p.u.. As a result, the SCC is reduced to 0.5 . Depending on the operating mode of the VSC, the $P-f$ and $Q-V$ droop gains are:

- Grid-supporting: $K_{\mathrm{pf}}=10$ and $K_{\mathrm{q}}=0.2$

- Grid-feeding: $\quad K_{\mathrm{pf}}=0$ and $K_{\mathrm{q}}=0$

The VSC, in grid-supporting mode, participates in the regulation of the $\mathrm{AC}$ voltage and frequency by controlling the active and reactive power delivered to the grid. On the other hand, the VSC, in grid-feeding mode, delivers constant power equal to $P_{\text {ref }}$ to the grid and regulates the reactive power to keep the terminal voltage equal to $V_{\text {ref }}$.

1) Dynamic limit of maximum power transfer capability of grid-supporting VSC: The evolution of the VSC active power can be seen in Fig. 7. As shown in Fig. 6 and mentioned above, the maximum transfer capability of the VSC, in steadystate condition, is approximately equal to the steady-state stability limit $\left(P_{\text {ref }} \approx 0.5\right.$ p.u.) defined by (24). However, in case of a sudden SCC, the maximum transfer capability of the VSC is determined by the dynamic limit [5]. That implies that if the pre-fault power operating point is higher than the dynamic limit, VSC will lose its synchronism and

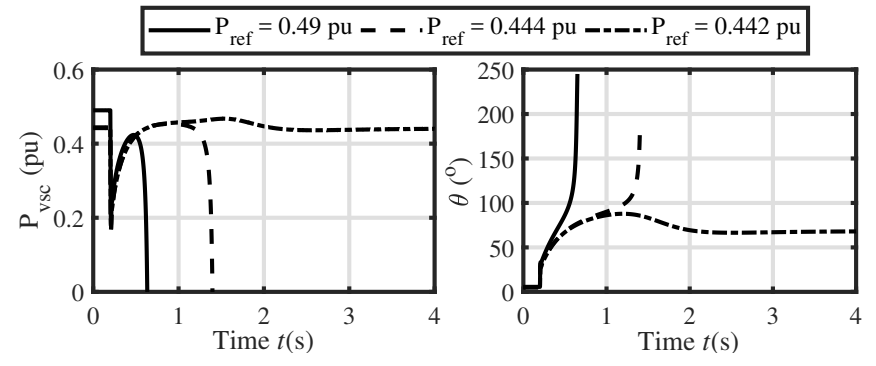

Fig. 7. Case 1 - Dynamic limit of maximum power transfer capability of grid-supporting VSC for $K_{\mathrm{pf}}=10$

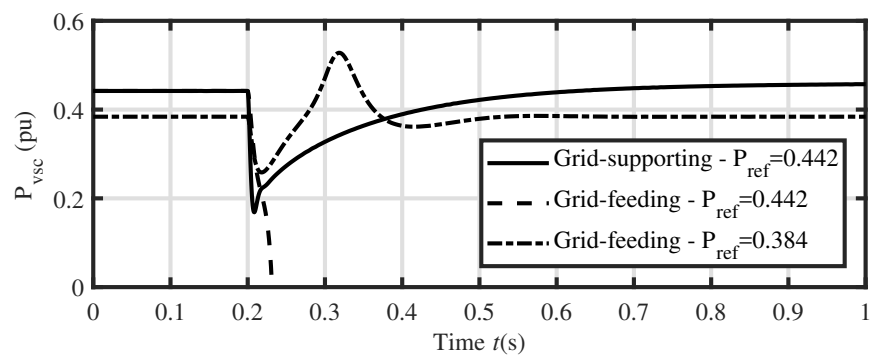

Fig. 8. Case 1 - Dynamic limit of the maximum power transfer capability of grid-feeding VSC

becomes unstable. This can be explained by the fact that, VSC prioritizes the active power and tries to restore it to its pre-fault value. However, due to the voltage dip, the maximum transfer capability temporarily decreases and instability occurs, due to the VSC trying to restore active power injection faster than the voltage is being restored.

As it can be seen in Fig. 7, VSC loses syncrhronism after tripping the short reactance, when the pre-fault power operating point is equal to the steady-state stability limit determined by (24). To identify the dynamic limit, the prefault power operating point is gradually decreased. As it is shown in Fig. 7, the first stable operating point was found to be $P_{\text {ref }}=0.442$ p.u..

2) Dynamic limit of maximum power transfer capability of grid-feeding VSC: The evolution of the VSC active power can be seen in Fig. 8. As before, to identify the dynamic limit for the grid-feeding converter, the $P_{\text {ref }}$ is gradually decreased. It can be seen in Fig. 8, that the dynamic limit for the gridfeeding converter is $P_{\text {ref }}=0.384$ p.u.. Thus, the dynamic limit of the grid-feeding VSC is lower.

3) Comparison between grid-supporting and grid-feeding VSC: Fig. 9 demonstrates a comparison between the gridfeeding and the grid-supporting scheme, where the initial operating point is equal to $P_{\text {ref }}=0.4$ p.u.. The dashed lines correspond to the grid feeding and the solid to the gridsupporting.

As shown in Fig. 9a, due to the frequency droop characteristic of the grid-supporting VSC, the power is reduced to 0.2 p.u. right after the disturbance. That leads to smaller voltage depression, so that $P_{\max }^{\mathrm{dyn}}>0.5$ p.u., even after the disturbance.

In the case of the grid-feeding VSC, the voltage depression is higher (see Fig. 9b), since the VSC tries to inject the initial $P_{r e f}$ to the AC system. As a result, the $P_{\max }^{\mathrm{dyn}}$ decreases and 


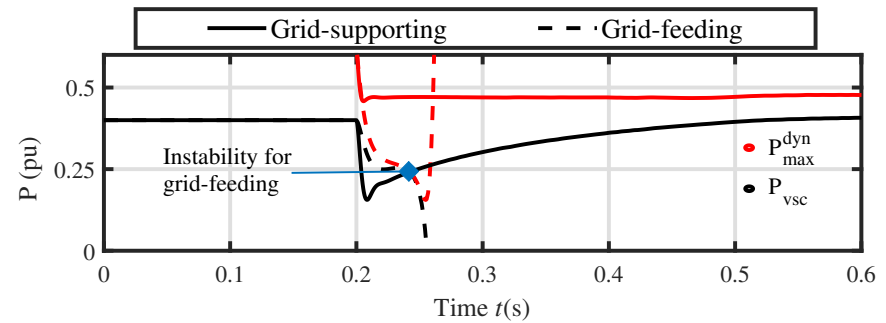

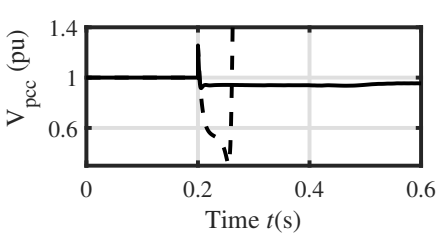

(b)

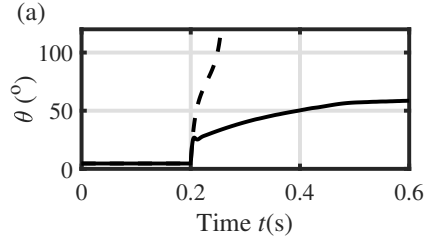

(c)
Fig. 9. Case 1 - (a) Maximum power transfer capability $P_{\max }^{\mathrm{dyn}}$ (red) and VSC active power (black), (b) PCC voltage, (c) Angle difference $\theta_{\mathrm{pll}}-\theta_{\mathrm{g}}$
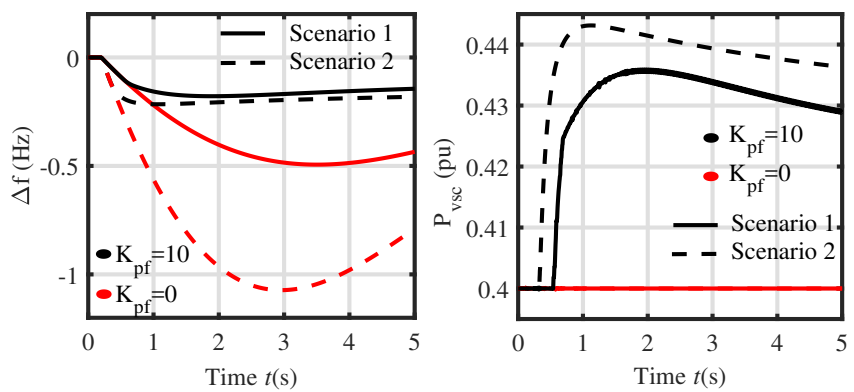

Fig. 10. Case 2 - Frequency deviation \& VSC active power, Comparison of frequency response with and without the frequency droop

becomes equal to $P_{\mathrm{vsc}}$ at $t=0.24 \mathrm{~s}$, which leads to the gridfeeding VSC losing synchronism (see Fig. 9c).

\section{B. Case 2 - Power imbalance for lower inertia}

The frequency deviation to a power imbalance is investigated for different levels of inertia. In this case, the grid impedance is considered equal to $X_{\text {eq }}=0.2$ p.u.. An increase of the load occurs at $t=0.2 \mathrm{~s}$. The considered disturbance is equal to $\Delta P_{\text {load }}=0.05$ p.u..

The evolution of the frequency is shown in Fig. 10, where the two presented scenarios in Section III.A were considered with and without the droop. The values for $K_{\mathrm{pi}}$ and $K_{\mathrm{pf}}$ are given in Table I. As it is depicted in Fig. 10, the impact of the frequency droop is mostly on the maximum frequency deviation, where it is significantly smaller compared to the case where the frequency droop is not considered. Although, the fast reaction of the VSC leads to reduced maximum frequency deviation, there is no direct contribution to the rate of change of frequency. As it was expected from Fig. 3, the frequency droop characteristic of the VSC contributes to the frequency stability and reduces the frequency deviation.

\section{CONCLusions \& Future WORK}

This paper has investigated the stability of a grid-supporting VSC connected to a weak AC grid. By means of eigenvalue analysis, the impact of $P-f$ and $Q-V$ droop characteristics of the VSC on the system stability is analyzed. Moreover, $\mathrm{H}_{2}$ norm is utilized to demonstrate how the outer loop parameters affect the AC frequency response. To gain further insight into the system properties, time domain simulations were performed for a sudden drop of SCC and a load disturbance.

The main findings are as follows:

1) In case of a grid-supporting VSC, due to the $P-f$ droop characteristic, the system synchronization can be preserved for larger disturbances of SCC.

2) The fast reaction of the VSC leads to reduced maximum frequency deviation. However, there is no direct contribution to the rate of change of frequency.

3) Unlike the effect of $P-f$ droop, the $Q-V$ droop characteristic reduces the dynamic limit of maximum power transfer capability of the VSC.

Future work is intended to investigate the interaction between the different parameters, characterizing the inertial and primary frequency control of the AC equivalent, for optimal tuning of $K_{\mathrm{pf}}$ and $K_{\mathrm{pi}}$, as well as, the influence of varying inertia levels on the dynamic limit of the VSCs.

\section{ACKNOWLEDGMENT}

This work is supported by the multiDC project funded by Innovation Fund Denmark, Grant Agreement No. 615400020B.

\section{REFERENCES}

[1] United Nations, "Paris Agreement," Tech. Rep., Dec. 2015.

[2] G. S. Misyris, S. Chatzivasileiadis, and T. Weckesser, "Robust frequency control for varying inertia power systems," in 2018 IEEE PES Innovative Smart Grid Technol. Conf. Europe (ISGT-Europe), Oct 2018, pp. 1-6.

[3] S. Chatzivasileiadis, D. Ernst, and G. Andersson, "The global grid," Ren. Energy, vol. 57, pp. $372-383,2013$.

[4] N. Flourentzou, V. G. Agelidis, and G. D. Demetriades, "VSC-based HVDC power transmission systems: An overview," IEEE Trans. Power Electron., vol. 24, no. 3, pp. 592-602, Mar. 2009.

[5] L. Papangelis, M. Debry, T. Prevost, P. Panciatici, and T. Van Cutsem, "Stability of a voltage source converter subject to decrease of shortcircuit capacity: A case study," in 2018 Power Systems Computation Conference (PSCC), Jun. 2018, pp. 1-7.

[6] J. Rocabert, A. Luna, F. Blaabjerg, and P. Rodríguez, "Control of power converters in AC microgrids," IEEE Trans. Power Electron., vol. 27, no. 11, pp. 4734-4749, Nov. 2012.

[7] J. Fradley, R. Preece, and M. Barnes, "VSC-HVDC for frequency support (a review)," IET Conf. Proc., pp. 1-6, Jan. 2017.

[8] L. Papangelis, X. Guillaud, and T. Van Cutsem, "Frequency support among asynchronous AC systems through VSCs emulating power plants," in 11th IET Int. Conf. on AC and DC Power Transmission, Feb. 2015, pp. 1-9.

[9] J. Huang and R. Preece, "HVDC-based fast frequency support for low inertia power systems," IET Conf. Proc., pp. 1-6, Jan. 2017.

[10] D. Gross, S. Bolognani, B. K. Poolla, and F. Dörfler, "Increasing the resilience of low-inertia power systems by virtual inertia and damping," in Proc. of IREP'2017 Symposium. Int. Institute of Research and Education in Power System Dynamics (IREP), 2017-08-31, p. 64.

[11] J. Z. Zhou, H. Ding, S. Fan, Y. Zhang, and A. M. Gole, "Impact of short-circuit ratio and phase-locked-loop parameters on the small-signal behavior of a VSC-HVDC converter," IEEE Trans. Power Del., vol. 29 , no. 5, pp. 2287-2296, Oct. 2014.

[12] J. A. Suul, S. D'Arco, P. Rodríguez, and M. Molinas, "Impedancecompensated grid synchronisation for extending the stability range of weak grids with voltage source converters," IET Gener., Transm. Dis., vol. 10, no. 6, pp. 1315-1326, 2016.

[13] T. Weckesser and T. Van Cutsem, "Equivalent to represent inertial and primary frequency control effects of an external system," IET Gener. Transm. Dis., vol. 11, no. 14, pp. 3467-3474, 2017.

[14] L. Shen, M. Barnes, R. Preece, and J. Milanović, "Frequency stabilisation using VSC-HVDC," in 2016 IEEE Power and Energy Society General Meeting, Jul. 2016, pp. 1-5. 